\title{
Vinculación y transferencia de sistema tecnológico para la reutilización de aguas grises
}

\author{
B. Garzónn ${ }^{1,3}$, L. Paterlini' ${ }^{1,3}$, G. Giuliano ${ }^{2,3}$
}

\section{Resumen}

Este trabajo tiene como objetivo exponer el proceso de vinculación y transferencia, de un sistema innovador tecnológico sustentable, que recupera las aguas grises proveniente de lavabos previo a su desecho, para reutilizarlas en depósito de inodoros. En el año 2015, el sistema fue diseñado y construido en Tucumán y difundido e implementado hasta la actualidad en Tucumán y Santiago del Estero. El mismo, tiene características de ser asequible y adaptable, por la posibilidad de instalarlo sin demasiadas intervenciones; por lo cual, es viable impactar en un gran sector de la población y en especial, en locales sanitarios de diferentes funciones edilicias de interés social (viviendas, escuelas, instituciones científico-tecnológicas, productivos, etc.). En el proceso de la investigación fue aplicado y se utilizó una combinación metodológica, que se enmarca en la Investigación Acción Participativa. Los resultados obtenidos fueron los siguientes: 1) Diseño y construcción del "Sistema ATRAG o Mochila "e"; 2) Desarrollo de "marca" y material e instrumentos para difusión, transferencia y adopción del sistema; 3) Acciones para la difusión de la vinculación, transferencia e implementación del sistema; 4) Generación de espacios para el debate y la concienciación de la problemática. En conclusión, se comprobó que

1Facultad de Arquitectura y Urbanismo de la Univ. Nac. de Tucumán - Secretaria Ciencia, Arte e Innovación Tecnológica 2Instituto de Estudios Ambientales y Desarrollo Rural de la Llanura Chaqueña, Universidad Nac. de Santiago del Estero; 3 Consejo Nacional de Investigaciones Científicas y Técnicas (CONICET), MInCyT; e-mail: bgarzono6@gmail.com; paterlinileonardo@gmail.com; gm.giuliano@gmail.com 
el análisis y la generación de los métodos de transferencia y sus recursos, son tan importantes como el proceso de diseño, construcción, instalación y monitoreo del funcionamiento del sistema en sí mismo. Se observó que en los edificios donde se implementó el sistema, se logró su integración apropiada y apropiable. Asimismo, fue positiva la generación de espacios para la promoción y concienciación sobre los beneficios del cuidado del ambiente, ya que en cada lugar, se inició un proceso búsqueda y transformación hacia la sustentabilidad.

Palabras clave: Sistema Innovador; Reutilización del Agua; Hábitat Sustentable.

\section{Abstract:}

The objective of this work is to expose the linking and transfer process of an innovative sustainable technological system that recovers gray water from sinks prior to disposal, to reuse them in a toilet tank. In 2015, the system was designed and built in Tucumán, and has been disseminated and implemented to date in Tucumán and Santiago del Estero. It has the characteristics of being affordable and adaptable, due to the possibility of installing it without too many interventions; therefore, it is feasible to impact a large sector of the population and especially, in sanitary premises of different social interest building functions (houses, schools, scientific-technological, productive institutions, etc.). In the applied research process, a methodological combination was used, which is part of the Participatory Action Research. The results obtained were as follows: 1) Design and construction of the "ATRAG System or Backpack e"; 2) Development of brand and material and instruments for dissemination, transfer and adoption of the system; 3) Actions for the dissemination of the link, transfer and implementation of the system; 4) Generation of spaces for debate and awareness of the problem. In conclusion, it was verified that the analysis and generation of the transfer methods and their resources are as important as the process of design, construction, installation and monitoring of the operation of the system itself. It was observed that in the buildings where the system was implemented, its appropriate and appropriable integration was achieved. Likewise, the generation of spaces for the promotion and awareness of the benefits of caring for the environment was positive, since in each place, a process of search and transformation towards sustainability began. 
Key words: Innovative System; Reuse of Water; Sustainable Habitat.

\section{Introducción}

El consumo mundial de agua ha aumentado por un factor de seis en los últimos 100 años y sigue creciendo constantemente a un ritmo de alrededor del $1 \%$ anual (AQUASTAT, 2010) con el aumento de la población, el desarrollo económico y los patrones de consumo cambiantes (UNESCO y ONU, 2020). El calentamiento global exacerbará aún más esta tendencia, ya que la demanda de agua tiende a aumentar con la temperatura (Gato et al., 2007). Esto ejercerá una presión significativa sobre las autoridades de agua para mantener el equilibrio entre la demanda y el suministro de agua (UNESCO y ONU, 2020). En el contexto mundial, América Latina y el Caribe frecuentemente es citada como una zona del planeta en la cual existe abundancia de recursos hídricos. En efecto, con una precipitación media anual de 1.600 milímetro, la región, concentra casi un tercio de los recursos hídricos mundiales. Sin embargo, su población equivale al $6 \%$ y su superficie al $13 \%$ de los totales mundiales. Ello significa que mientras su disponibilidad media de agua por habitante alcanza aproximadamente a 22 mil metros cúbicos por habitante por año, a nivel mundial dicho valor es de sólo un poco más de 6 mil. Argentina dentro de la región, entre 18 países ocupa el octavo puesto, con mayor disponibilidad hídrica por año y por habitante, con 26.000 (Mm3/año) y 20.500 ( $\mathrm{m}_{3} / \mathrm{hab} / \mathrm{año}$ ) respectivamente (BID y CEPAL 2018). Sin embargo, es uno de los países con menor nivel conciencia y de acciones por el cuidado del recurso hídrico.

En la actualidad, en nuestro país existen en el mercado numerosos sistemas que reutilizan aguas grises, que por su elevado costo, son de difícil acceso para la mayoría de la población, siendo acotado el alcance de los mismos y que por lo tanto, se limita su difusión para crear la concienciación necesaria, sobre la importancia de la protección de los recursos naturales. En la provincia de Tucumán, en el año 2015 se desarrolló el sistema "ATRAG" Alternativas de Tecnológicas para la Acumulación, Filtrado y Reutilización de Aguas Grises (designación del proyecto) o "Mochila e" (designación comercial). El sistema recupera aguas grises del lavabo y las lleva hacia el sistema de depósito de agua para inodoro (mochila) para proceder luego a su desagote a través del mismo. Es decir, se plantea una mochila de inodoro que trata el agua gris para luego ser utilizada en la evacuación de aguas negras del inodoro. Se 
presenta al sistema como un elemento innovador, de características apropiables por los potenciales usuarios y en cualquier medio donde se pretenda implementarlo; siendo también, de bajo costo por su material, resuelto en fibra de vidrio. La intención primaria ha sido generar un producto practicó para los usuarios, que involucre intervenciones constructivas mínimas y que se presente, como un objeto "reconocido”, por estética y funcionalmente, en el espacio baño (Garzón y Paterlini, 2016). Se diseñó y desarrolló la marca y el empaque del producto, su manual de instalación y uso; además de folletos, afiches y audiovisual para la difusión del mismo, que apuntan a mostrar y remarcan sus propiedades, como un sistema "accesible" por ser económico para los usuarios y "amigable" con el ambiente, en cuanto al ahorro de agua potable para consumo. Por otro lado, se generaron espacios de debate respecto de esta problemática, que es importante para poner en conocimiento a la población, sobre la posibilidad de desarrollo de sistemas con características similares. Este trabajo tiene como propósito: contribuir a la difusión, adopción e implementación de un sistema tecnológico innovador, para reutilización de aguas grises provenientes del lavabo, como aporte al cuidado de los recursos hídricos, para la sostenibilidad ambiental.

\section{Objetivos Generales}

1. Acercar a la población y especialmente al sector más vulnerable, un sistema sustentable y asequible, que reutiliza el agua de lavatorios para la carga de depósito de inodoros, mejorando las condiciones de higiene, salubridad, bienestar y confort.

2. Generar instancias de difusión, demostrando la conveniencia de la reutilización de aguas grises.

3. Lograr la adopción e implementación del prototipo sustentable, integrando los requerimientos y condicionantes ambientales, culturales, productivos, sanitarios, etc.; de los sitios en consideración.

\section{Objetivos Específicos}

1. Desarrollar y construir el sistema y su empaque, con una gráfica atractiva, que comunique sus ventajas, en relación a un mismo producto del tipo convencional.

2. Elaborar el manual de instalación y uso, para su distribución comercial, poniendo a los usuarios en conocimiento, del mejor modo de colocación y utilización del mismo. 
3. Diseñar herramientas gráficas y audiovisuales para la difusión sobre la concientización respecto a la necesidad de hacer un uso racional del agua.

4. Generar la vinculación con diferentes instituciones, para la transferencia y apropiación del sistema en edificaciones de interés social, mejorando la situación ambiental y la calidad de vida de los usuarios.

\section{Metodología}

Se utilizó una combinación metodológica que se enmarca en la Investigación-Acción Participativa. Para ello, se emplearon los siguientes métodos: exploratorio, analítico-descriptivo, experimental y el estudio de casos. En una primera etapa, se realizó la búsqueda de antecedentes de la temática, el análisis de propuestas de diseños y su desarrollo. Seguidamente, se articuló con micro-emprendedores y especialistas en construcción, para la selección de la alternativa superadora. A la misma, se le realizó sucesivas evaluaciones para su ajuste, en las etapas de experimentación logradas: en gabinete, laboratorios y en campo. Seguidamente, se ejecutaron prototipos del sistema de reutilización de aguas grises el proceso constructivo con cooperativas, y se mejoró su funcionamiento para uso intensivo. Se diseñó y materializó el "packaging” y el manual de colocación, uso y mantenimiento.

La segunda etapa, se inició realizando la selección y vinculación con las Instituciones para la transferencia del sistema, coordinando con técnicos y profesionales responsables de la producción de viviendas sociales y edificios de Tucumán (IPVyDU y CCT) y de escuelas en Santiago del Estero (DGA); articulando en conjunto, la elaboración de documentación técnica específica a cada implementación. Posteriormente, se concretó las transferencias de los sistemas, en locales sanitarios de los establecimientos seleccionados en ambas provincias, previo asesoramiento y capacitación a técnicos y comunidades según necesidades inherentes. Finalmente, se continuó con la difusión de los análisis y las propuestas, en publicaciones y exposiciones de reuniones científico-tecnológicas, mediante diversas herramientas: maqueta a escala real, folletos, afiches y audiovisuales explicativos, etc.; para la concienciación de la problemática y de las soluciones alternativas sustentables para mejorar la calidad de vida y el hábitat. 


\section{Resultados}

\section{Diseño y construcción del Sistema ATRAG}

El desarrollo tecnológico se basó en el diseño y construcción de un depósito de agua para inodoro tipo "mochila" de fibra de vidrio, que recibe las aguas grises del lavabo (ver Figura $N^{o}$ 1). En su interior, el dispositivo cuenta con un sistema de filtrado que se realiza primeramente, donde decantan las materias sólidas que pudiese traer el agua. A continuación, el agua gris se deposita dentro de la mochila, la cual cuenta con un sistema de potabilización y tratamiento de la misma, previendo que el agua podría estar dentro del depósito hasta varios días en reposo, evitándose así la producción y reproducción de bacterias y gérmenes que son nocivas para el ser humano y que podrían propagar malos olores (Daives et al., 2019). El dispositivo tiene una descarga dual, es decir de doble volumen, el cual al presionar uno u otro botón libera -según la necesidad de descarga- entre 9 y 12 litros, logrando así también un uso racional del agua reutilizada (Figura 1). Es de destacar el hecho que en el caso que no hubiere utilización del lavabo por un período de tiempo, se prevé una conexión a la red para ingreso de agua desde red para el depósito de agua. De la misma forma, si hubiere un exceso en la utilización de agua del lavabo, el dispositivo cuenta con un rebalse interno, evitando el sobrellenado de la "mochila e". Todos los elementos accesorios mencionados, e inclusive la construcción del sistema son de bajo costo, como así también, el mantenimiento de la misma. Estas fue la premisa principal que guió el diseño y desarrollo del artefacto, para lograr un producto apropiado y apropiable para su implementación.

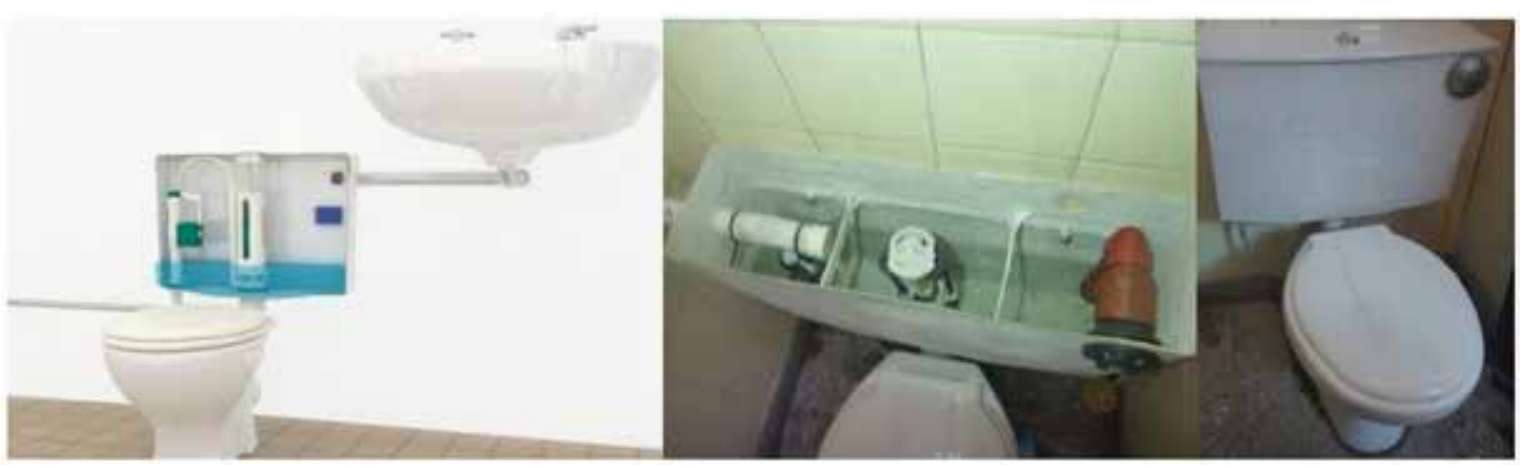

Figura $N^{o}$ 1: Fotorrealismo del diseño y fotografías, materialización e instalación del Sistema ATRAG. Fuente: Elaboración propia y fotografías tomadas por los autores. 


\section{Desarrollo de "marca" y material e instrumentos para la difusión,} transferencia y adopción del sistema

La construcción de la marca, está estrechamente ligada al producto que se quiere vender y la manera de acercar el mismo al público. El nombre comercial, elegido para el depósito de agua es "e“, pues está relacionado con: “ecológico, económico y estético”. Esto último relacionado, con el hecho de tratarse de un objeto que ya se encuentra visual, constructiva y formalmente integrado en el espacio baño y que no es necesario adicionar ningún otro, para su normal funcionamiento (ver Figura 2). El empaque fue trabajado de manera tal, que utilice materiales de bajo costo, reciclables y reutilizables. Un diseño tipo “maletín”, con toda la gráfica incorporada, de líneas simples y mostrando el sistema, su funcionamiento y los beneficios de cuidar el agua, buscando de concientizar al respecto.
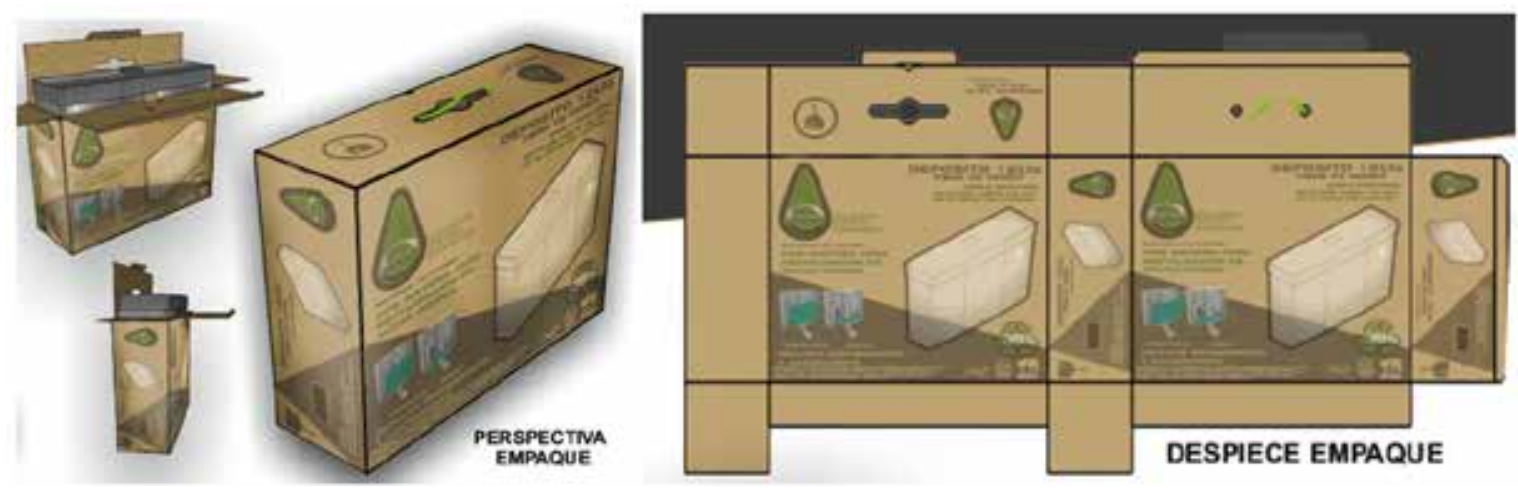

Figura $N^{o}$ 2: Diseño del empaque plegado y desplegado. Fuente: Realizado por el equipo de investigación.

La folletería que acompaña al dispositivo, es explicativa de lo necesario de consumir racionalmente el agua y de por qué el sistema es beneficioso, con su uso y se impone sobre otros que puedan ser de similares características (Figura $N^{o} 3$ ).
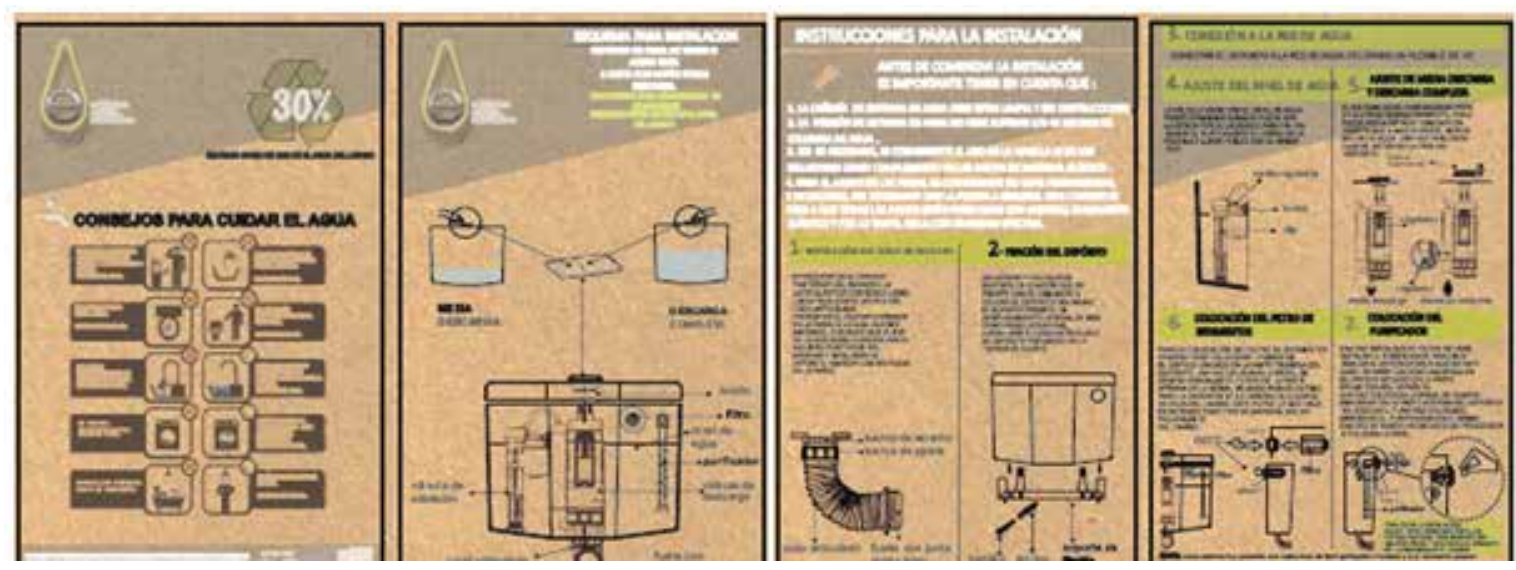

Figura $N^{o}$ 3: Diseño de folleto de instrucciones de instalación. Fuente: Realizado por el equipo de trabajo. 


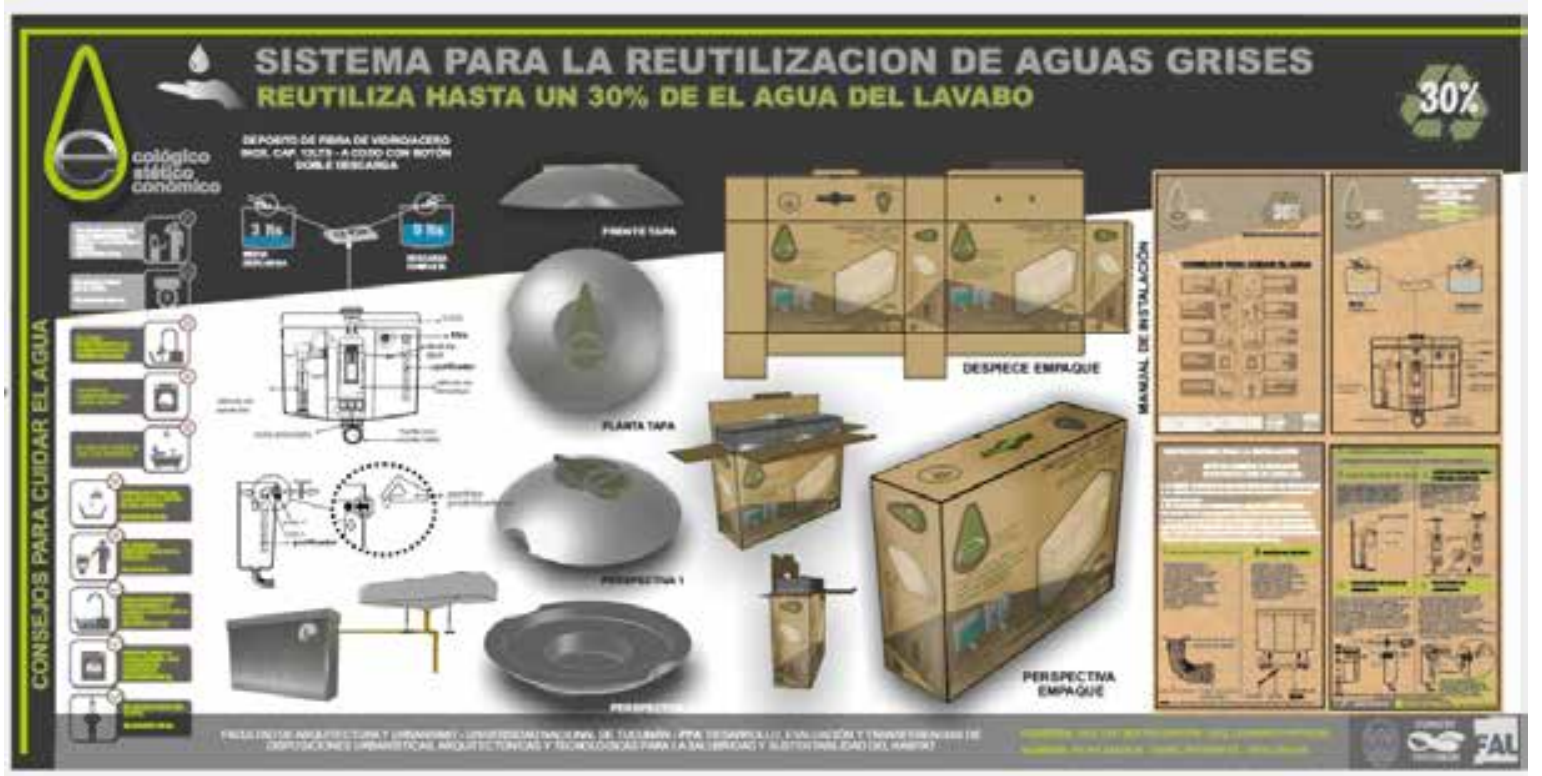

Figura $N^{o}$ 4: Afiche de exposición del sistema para difusión. Fuente: Realizado por el equipo de trabajo.

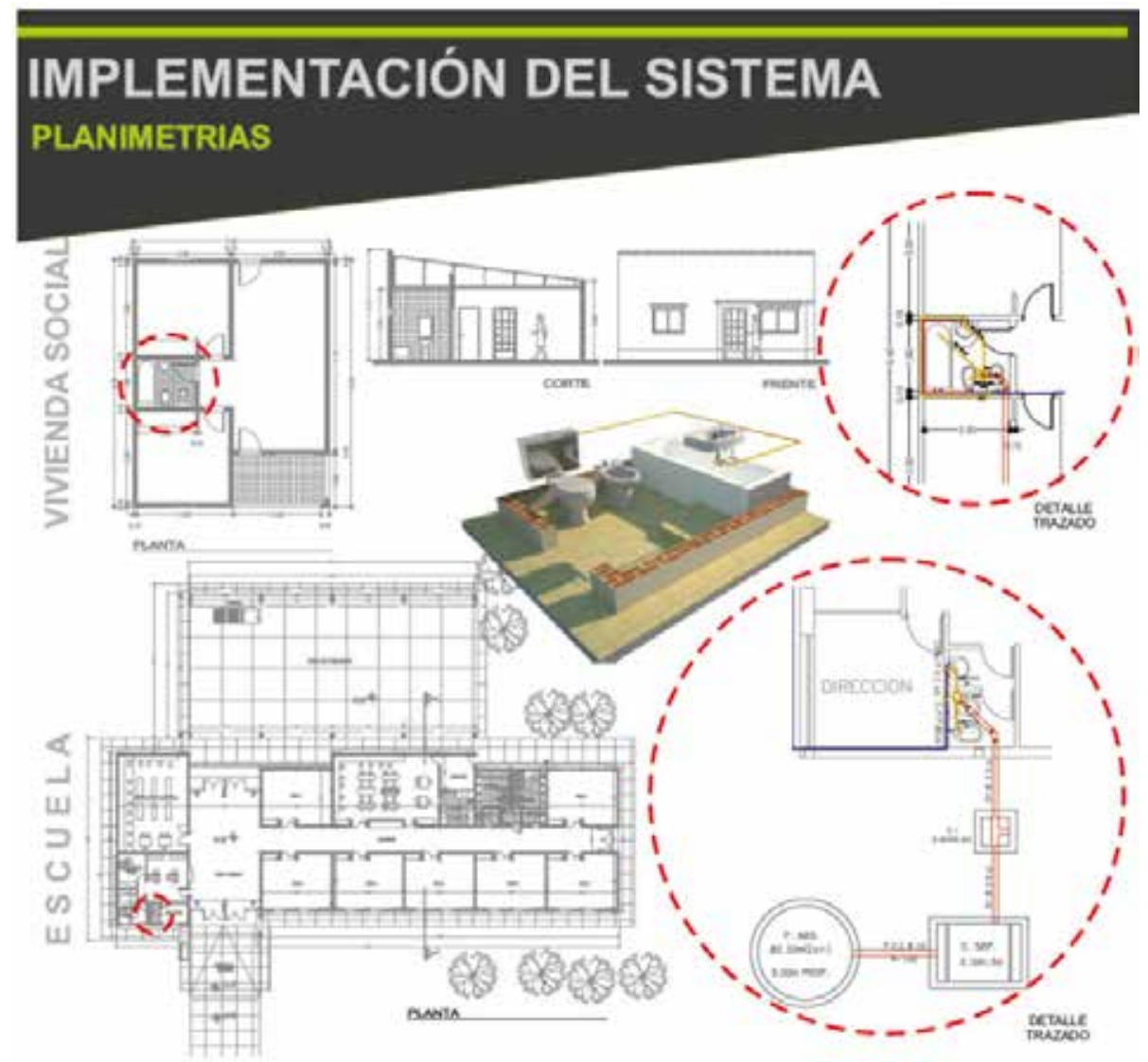

Figura $N^{o}$ 5: Afiche para la implementación. Fuente: Elaboración propia.

El corolario de la puesta en diseño del isologotipo (símbolo gráfico y tipográfico) de la "marca", es el afiche para de exposición. El mismo, se estructura tomando los puntos más generales de relato sobre el uso racional del agua, la descripción del 
sistema en general, su funcionamiento, las partes que lo conforman, el por qué de la “imagen", el empaque y su folletería para difusión (Figura $N^{o} 4$ ). Además, se diseñaron afiches donde se muestran las posibilidades de incorporación del sistema, tanto en edificios existentes como en nuevos, con distintas funciones, envergadura, analizando hasta nivel de detalle, en 2 y 3 dimensiones (Figura $N^{o} 5$ ).

\section{Acciones y Herramientas para difusión de la Problemática, Vincu- lación, Transferencia e Implementación del Sistema}

\subsection{Implementación en Viviendas Sociales de Tucumán}

Un eje importante como soporte del proyecto, fue la aplicación del sistema en viviendas sociales en el marco de la producción estatal. Se coordinó con uno de los programas habitacionales que se desarrollan desde el Instituto de la Vivienda y Urbanismo (IPVyDU) de Tucumán, para su implementación y evaluación. Se seleccionaron dos viviendas para instalar el sistema y que contribuyan a la salubridad y sustentabilidad de su hábitat y de sus usuarios (Figura $N^{\circ} 6$ ). Se realizó un estudio de factibilidad de la implementación, planteando la incorporación del sistema en el espacio sanitario, con lo cual, se realizó planimetría completa (Figura $N^{o} 7$ ) para tal fin, como detalles constructivos, croquis, etc.. Algunas consideraciones de tales tareas, son las que se detallan a continuación: 1. La incorporación del sistema en los espacios sanitarios se debe plantear desde el inicio del proceso de diseño de los mismos (caso de Vivienda de Aguilares, Río Chico, Tuc.); 2. La implementación en viviendas construidas también es posible, con un bajo costo al momento de tener
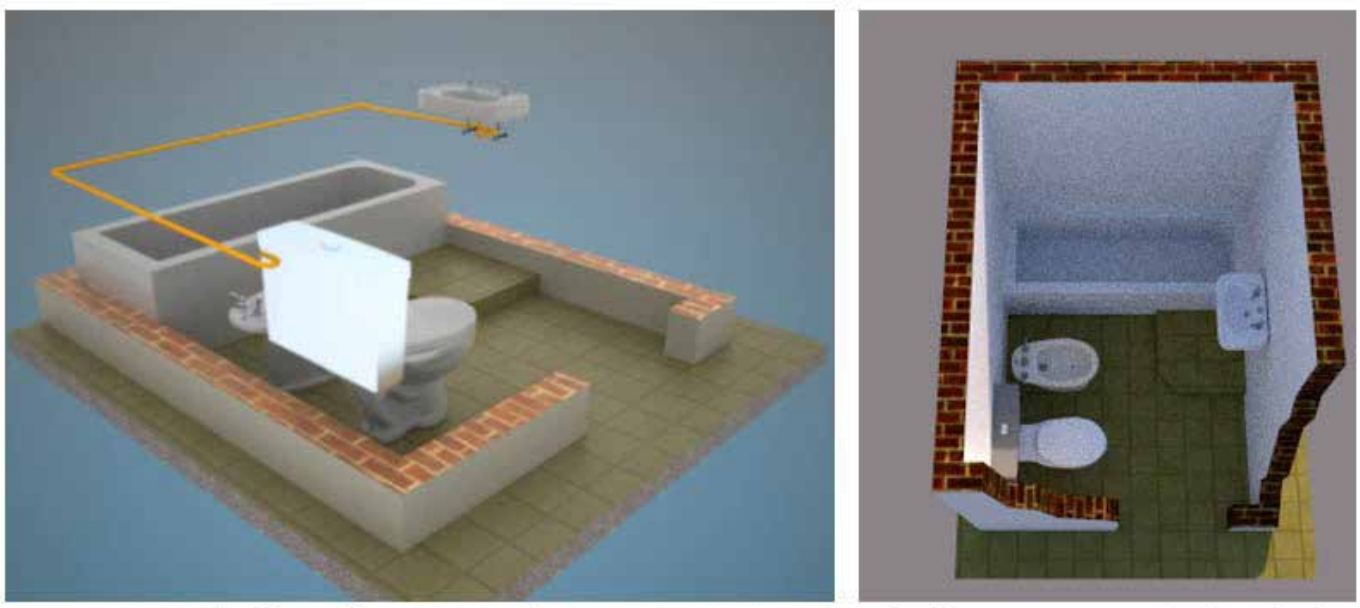

Figura $N^{\circ}$ 6: Imagen $3 D$, para la implementación en vivienda de interés social. Vivienda en Localidad de Aguilares, departamento Río Chico, Provincia de Tucumán. Fuente: Realizado por el equipo de trabajo. 
que realizar modificaciones del espacio existente y en la instalación del artefacto (caso vivienda Los Ralos, Cruz Alta., Tuc.) . En esta comunidad, se desarrollaron reuniones informativas y talleres de capacitación para su apropiación e instalación (Figura $N^{\circ} 8$ ).
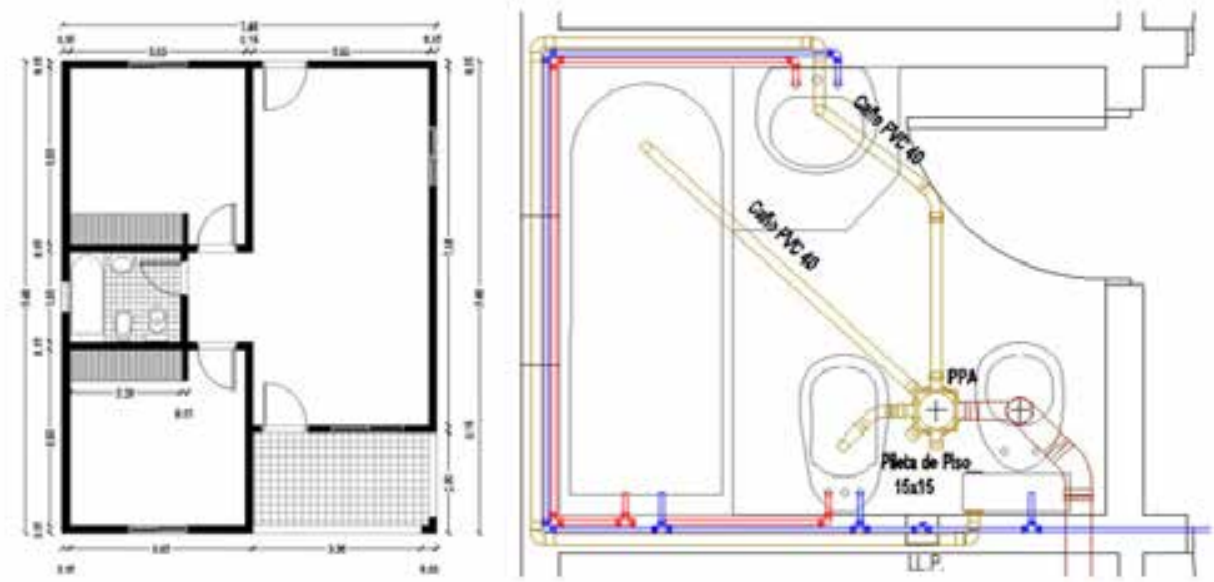

Figura $N^{o}$ 7: Planta de Arquitectura en Vivienda construida en Los Ralos, Cruz Alta, Tucumán y detalle de adecuación de, trazado de la instalación sanitaria para implementar Mochila "e". Fuente: Elaboración Propia.
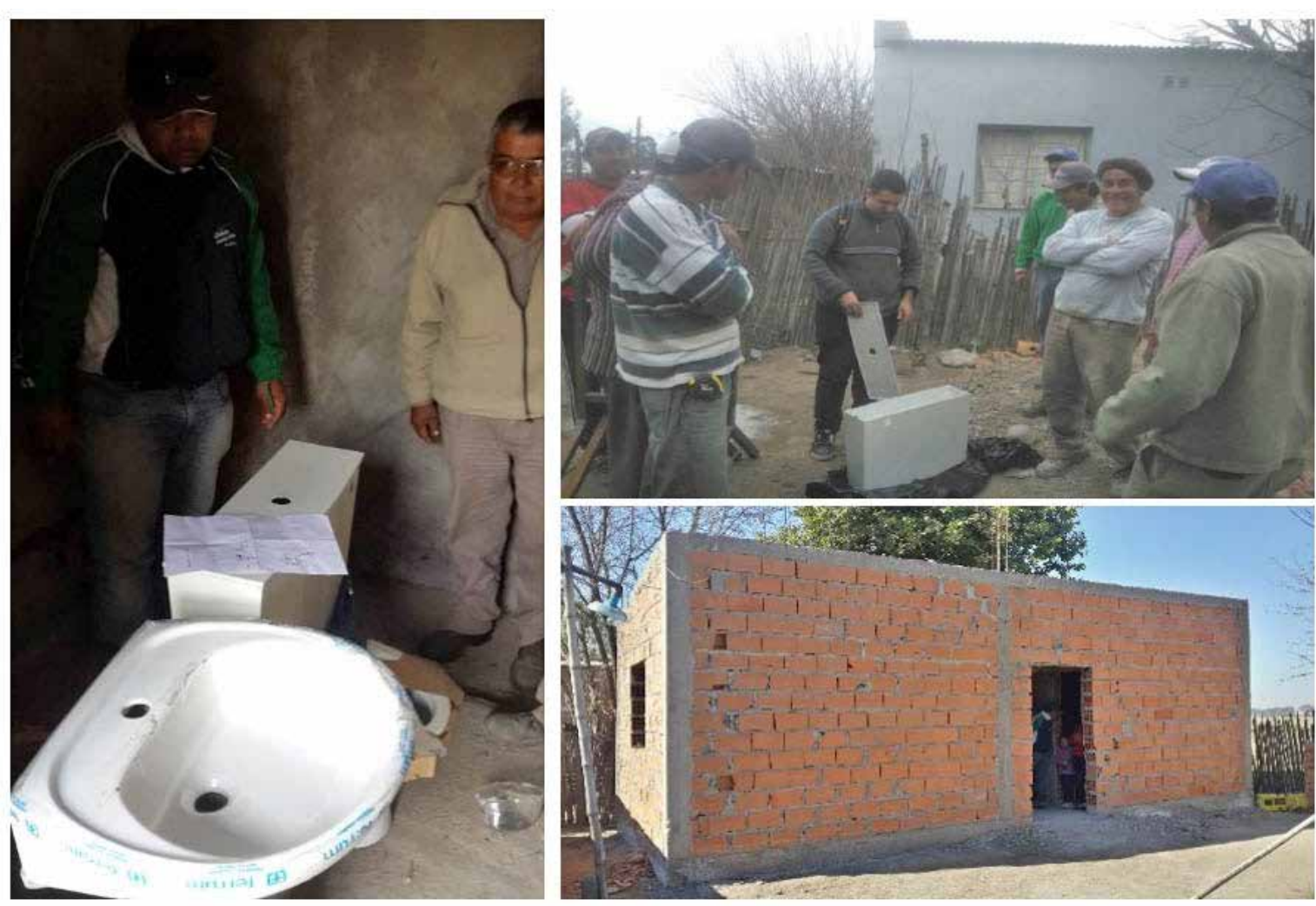

Figura No8: Fotografías de la implementación en vivienda social de los Ralos, Cruz Alta, Tucumán. Fuente: Realizado por el equipo de trabajo. 


\subsection{Implementación en escuelas rurales y urbanas de Santiago del}

\section{Estero}

En el año 2016, se realizó la vinculación con la Dirección General de Arquitectura de Santiago del Estero, responsable de la construcción y el mantenimiento de escuelas estatales. Así se concretaron, instancias de transferencia, instalación y monitoreo de sistemas, en baños en refacción total de escuelas rurales y en existentes, de escuelas urbanas donde, hubo que realizar las modificaciones pertinentes. En escuelas del interior, donde el agua es un recurso escaso; se buscó casos con sanitarios que estaban en obra de manera integral y como parte de la política escolar de "educar-haciendo" se instalaron las "mochilas e", los cuales siguen funcionando correctamente, hasta la actualidad (Figuras 9 y 10). Los sanitarios escolares urbanos, se convirtieron también en "modelos demostrativos" para sus comunidades escolares (Figura $N^{o} 11$ ). A fines del 2020, se instalará otra "mochila e", en sanitario de escuela rural en terminación (Figura $N^{\circ} 12$ ).
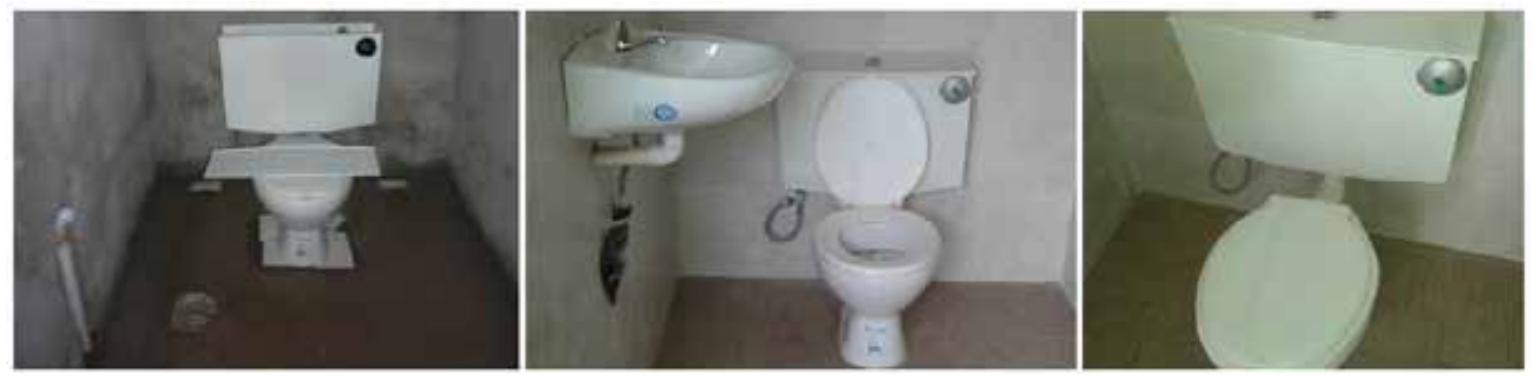

Figura $N^{\circ}$ 9: Sistema implementado en sanitario de docentes, en Escuela $N^{o} 923$ localidad "El Hoyón", Departamento Atamisqui, Provincia de Santiago del Estero. Fuente: Equipo de Investigación.
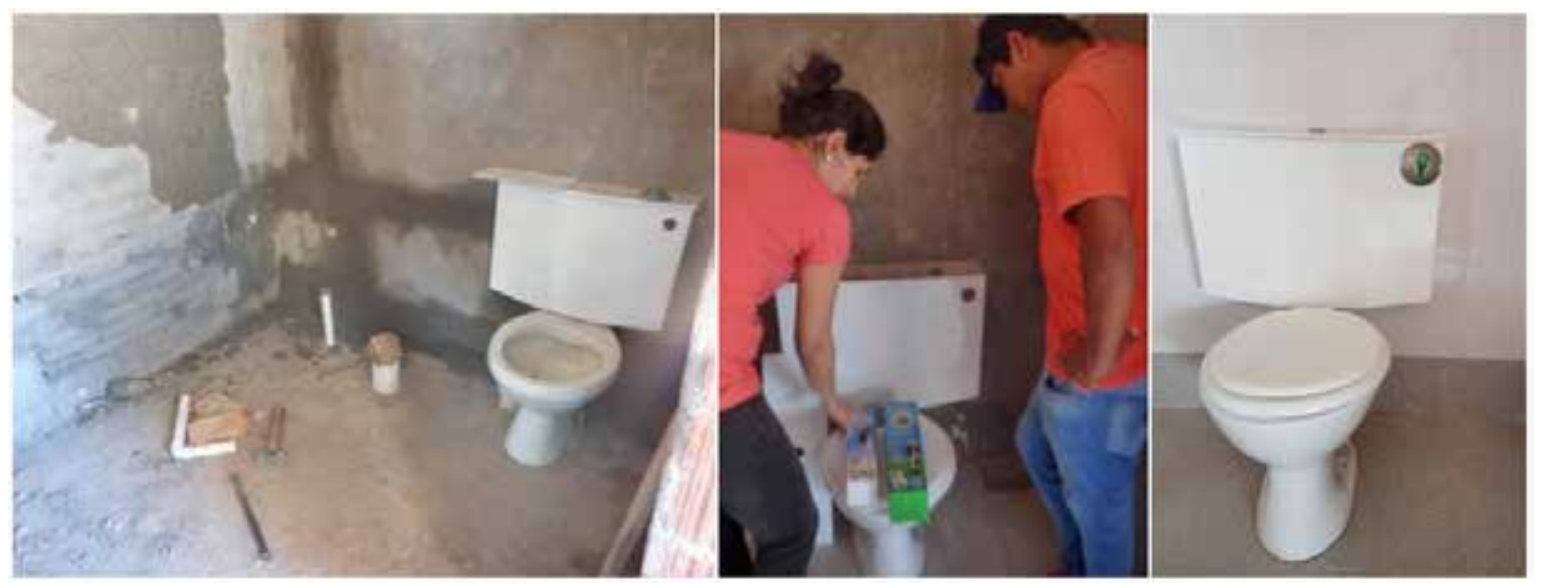

Figura $N^{o}$ 10: Escuela rural, sistema implementado en sanitario docentes, en Escuela $N^{\circ} 71$, Loc. La Cañada, Departamento Figueroa, Provincia de Sgo. del Estero. Fuente: Equipo de Investigación 


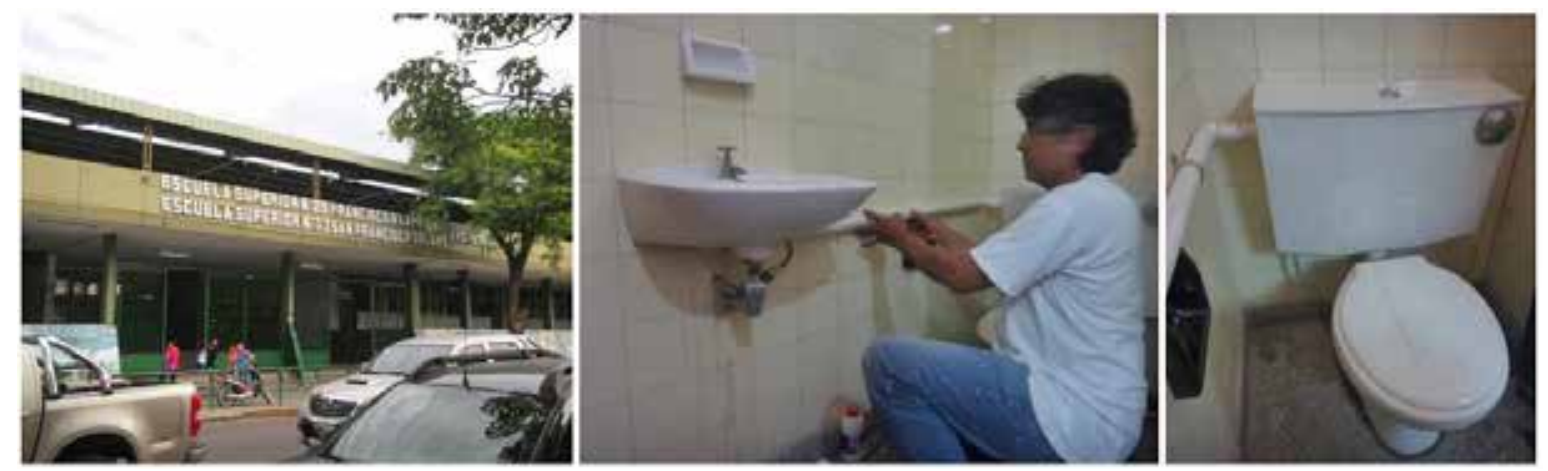

Figura $N^{\circ} 11$ : Sistema implementado en sanitario de dirección, en escuela estatal urbana $N^{o_{29}}$ "Francisco Laprida", ciudad capital de Santiago del Estero. Fuente: Fotografías tomadas por el equipo de trabajo.
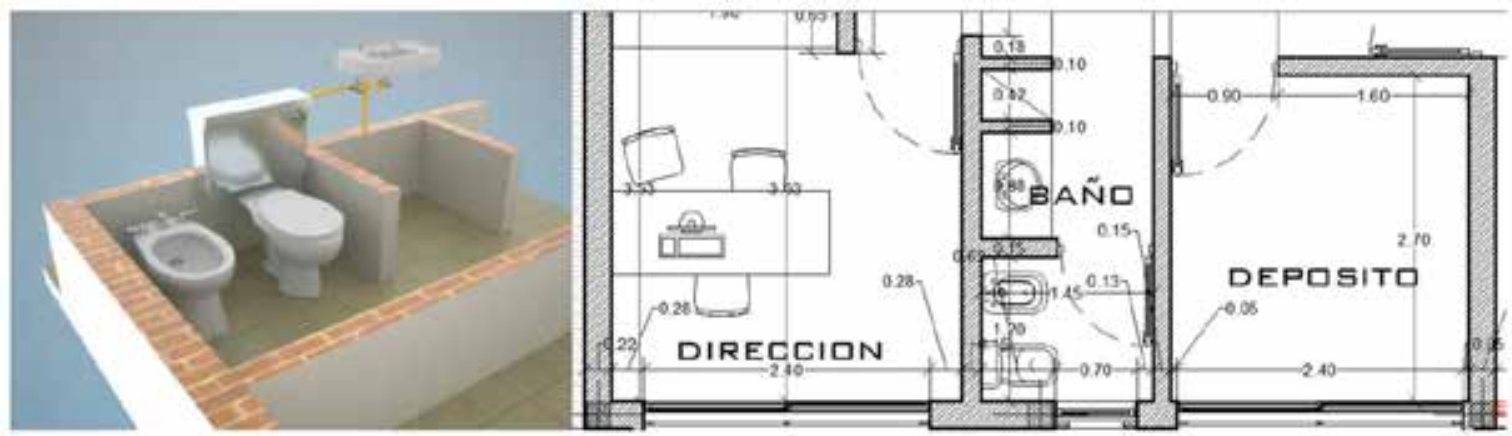

FFigura $N^{o}$ 12: Estudios de futura implementación de "mochila e", en núcleo sanitario docentes, de Escuela Secundaria de Vilmer en construcción, Robles, Santiago del Estero. Fuente: Elaborada por los Autores.

\subsection{Instalación del sistema en núcleo sanitario del CCT Conicet}

\section{Tucumán.}

Por último, se colocó el sistema en el núcleo sanitario del Centro CONICET de Tucumán. La experiencia se concretó, gracias a la invitación por parte de las autoridades para instalar el producto, resultado de trabajo alcanzado por investigadores CONICET. El sistema fue implementado en un baño existente, con necesidad de reparaciones y ante la posibilidad de colocar un sistema innovador y de características sustentables, optaron por el mismo. Como proceso de la instalación, se realizaron planimetrías del núcleo sanitario y señalética, donde se indica que el baño cuenta con el producto innovador (Figura $N^{o} 13$ ). Además, se procedió a la capacitación del sanitarista, que trabajó en la implementación del sistema y del personal de la institución, por su mantenimiento. 

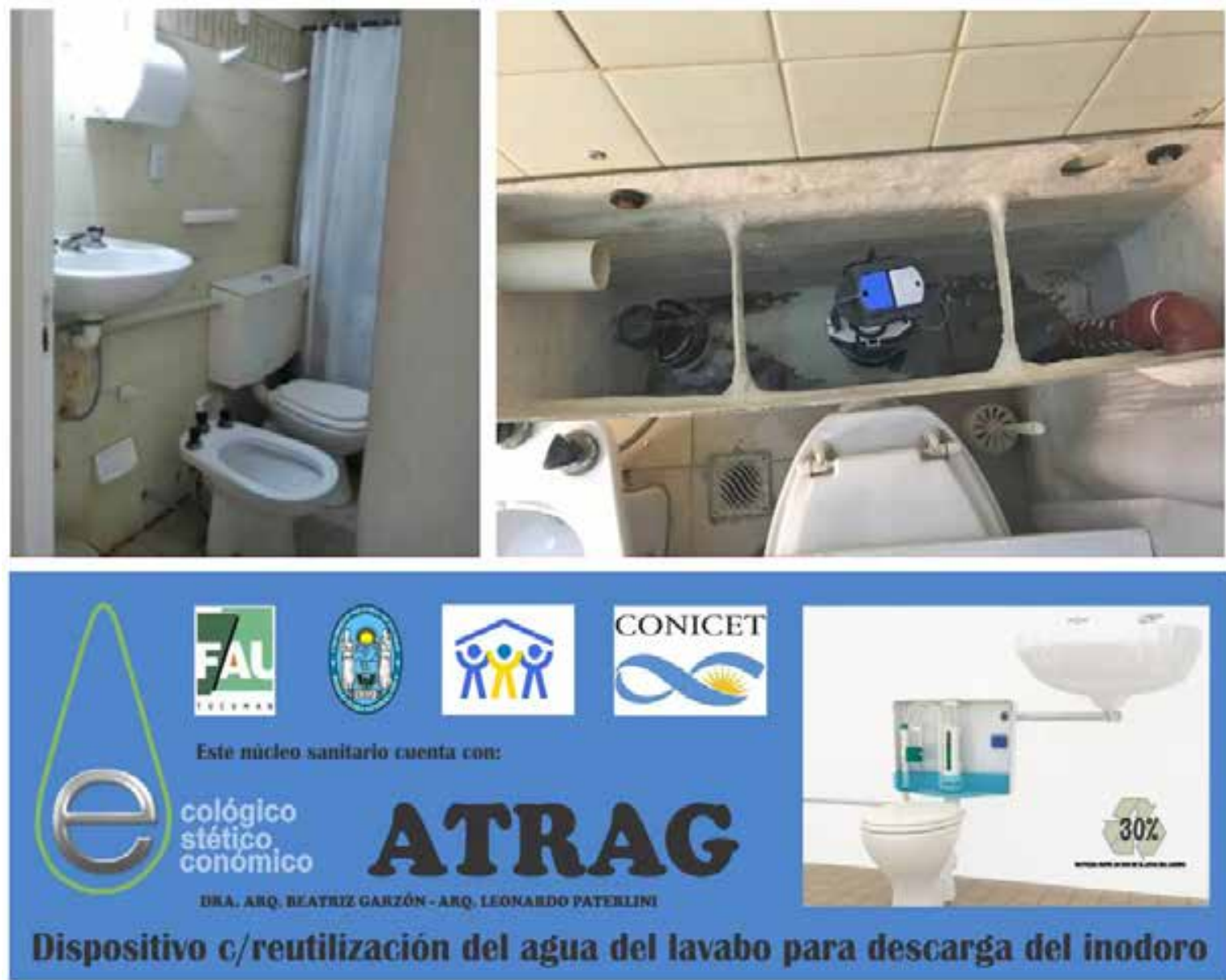

Figura $N^{\circ}$ 13: Sistema ATRAG instalado en el núcleo sanitario del CCT Tucumán y diseño de señalética. Fuentes: Fotografía tomadas por el equipo de trabajo e imagen de elaboración propia.

\section{Generación de espacios para la difusión, transferencia y debate}

Una vez diseñada y desarrollada, la información del sistema y aprobada su elaboración, se procedió a exponer y promover el sistema, a fin de poner en conocimiento a la población general y a la académico-científico-tecnológica, técnica y profesional, en particular, sobre las nuevas propuestas para el mejoramiento habitacional y el cuidado del ambiente. Así también, se buscó acercar a los usuarios, la comunicación y el debate sobre la problemática del agua, su utilización racional y la posibilidad de reutilización de aguas grises.

4.1. Transferencia "Feria Expo 2015" en Tucumán, en el Stand de CONICET

En San Miguel de Tucumán, en la Feria EXPO del año 2015 y dentro del espacio de CONICET, se expuso el dispositivo de recuperación de aguas grises. Para tal fin, 
se preparó una maqueta en escala 1:1, para simular el real funcionamiento del sistema. La maqueta cuenta con lavabo, inodoro y conexiones de cañerías, a la vista en falsa pared de madera y el dispositivo "mochila e", con el sistema de filtrado en su interior. La simulación del sistema, atrajo la atención de numerosas personas que se asombraron por la simpleza del dispositivo y por la considerable reducción del 30\% del consumo del agua, con la utilización del mismo. Entre el público, hubo gran cantidad de estudiantes de escuelas primarias, constructores, sanitaristas, emprendedores, familias, etc. Se abordó el tema del consumo racional de agua, problemáticas ambientales generales, tecnologías alternativas, la necesidad de buscar propuestas para alcanzar la sostenibilidad a largo plazo, etc. Además, se proyectó un video animado, el cual explica el funcionamiento del sistema y los beneficios que apareja su uso (Figura $\mathrm{N}^{\mathrm{o}} 14$ ). La exposición tuvo excelente aceptación, siendo el stand de CONICET mencionado en los diarios locales como un espacio para el acercamiento, el juego y el conocimiento.

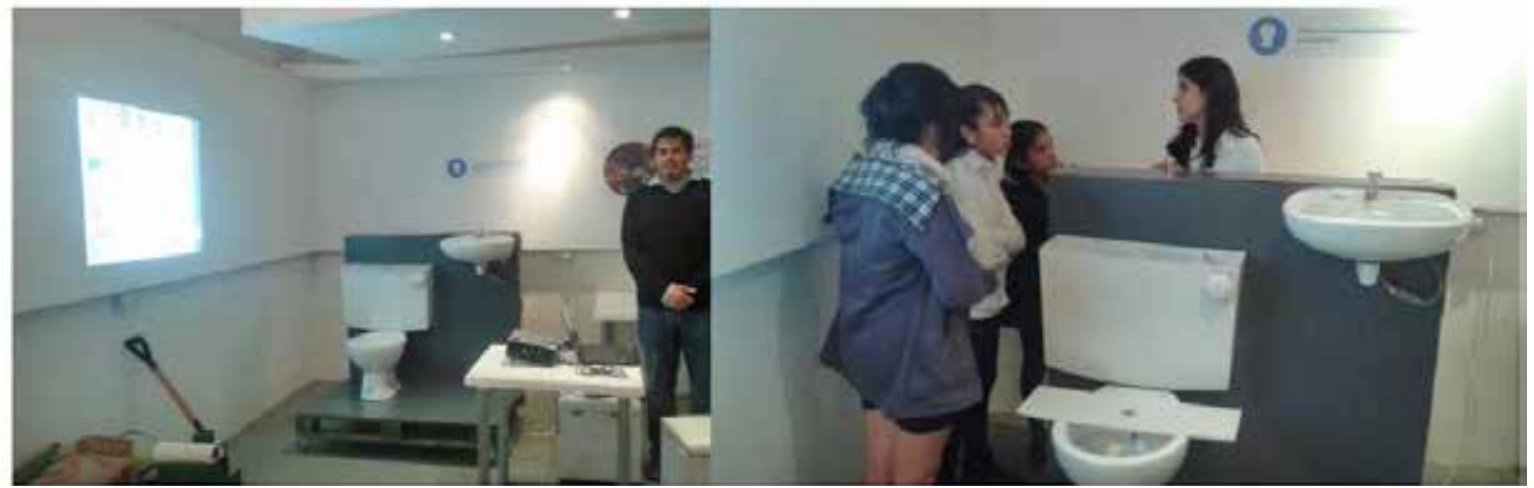

Figura $N^{\circ} 14$ : Feria Expo 2015, Stand CONICET: audiovisual en curso y maqueta 1:1 para difusión e intercambios con el público. Fuente: Fotografías tomadas por los autores.

\subsection{Muestra en el hall de la Facultad de Arquitectura y Urbanismo}

En el año 2016, se realizó una muestra en el Hall de la Facultad de Arquitectura y Urbanismo de la Universidad Nacional de Tucumán, en la cual se mostraron todos los elementos que componen al sistema: la mochila, piezas sanitarias. Además, de lo anterior, se presentó el empaque, el isologotipo, manual y folletos. Junto a ello, también el afiche explicativo, del funcionamiento del sistema, su instalación y los datos cuantitativos, de los valores de notables reducción del consumo de agua, por cada núcleo sanitario y tipo edilicio, al momento de su utilización. El sistema se expuso en un área junto con otras investigaciones sobre disposiciones tecnológicas, arquitectónicas y urbanísticas sustentables (Figura $N^{\circ} 15$ ). 


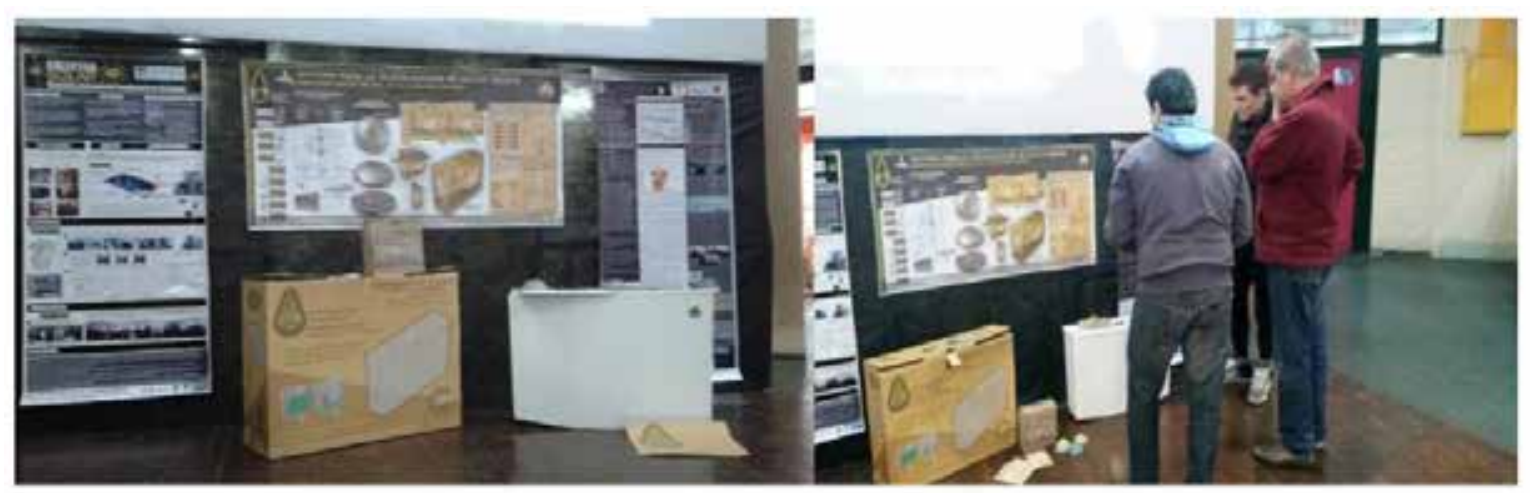

Figura $N^{0_{15}}$ : Muestra en la Facultad de Arquitectura de la Universidad Nacional de Tucumán. Fuente: Fotografías tomadas por los autores.

El sistema suscitó la atención, el interés y aceptación entre los presentes. Por lo que, se observa recurrentemente, la predisposición que existe en el medio para seleccionar y apropiarse de nuevos sistemas, por ser sustentables. Lo cual, manifiesta lo importante que es la problemática del uso racional del agua en la actualidad y lo poco abordado en nuestras agendas. Surgieron, además, inquietudes y comentarios, como la actual situación de colocación de medidores en las viviendas urbanas, situación que obligadamente lleva al usuario a un uso más cuidado del agua; poner en conocimiento y aplicar métodos y alternativas para el ahorro de recursos; acercar tales tecnologías a un usuario, aun cuando sea descuidado al respecto. Asimismo, se proyectó un video animado, el cual explica el funcionamiento y los beneficios que apareja el uso de tal sistema.

\section{Conclusión}

El sistema ha sido bien recepcionado y adoptado por los usuarios de los diferentes edificios de interés social, que se alcanzaron a través de su vinculación y transferencia: en viviendas y CCT Tucumán y en escuelas rurales y urbanas de Santiago del Estero. Fue comprobado, que el análisis y la generación de los métodos de transferencia y sus recursos, son tan importantes como el proceso de diseño, construcción, instalación y monitoreo del funcionamiento del sistema en sí mismo. Se determinó que el acercamiento del dispositivo tecnológico sustentable a los usuarios, se logra mediante instancias de transferencias adecuadas y apropiadas. Además se observó, que existe poca información en la población al respecto de la implementación de dispositivos sustentables, que permiten colaborar con la preservación del ambiente; sin embargo, con las difusiones y capacitaciones 
realizadas, se logró crear coincidencia e interés en cada comunidad. Por lo tanto, se corroboró que existe la necesidad en el medio, de que se continúe trabajando con la temática en profundidad, para mayor producción y apropiación de soluciones alternativas en el cuidado del agua, como recurso vital y de otros tipos sistemas ambientalmente amigables y que, a la vez, mejoren la calidad de vida de la sociedad y en especial, de los sectores más vulnerables.

\section{Agradecimientos}

A todos los actores participantes, al CCT Tucumán, por la disposición y apertura para la instalación de la tecnología.; al equipo de la Secretaria de Estudios y Proyectos de la DGA de Santiago del Estero, por colaborar en la coordinación para difusión y trasferencia del sistema en las escuelas; y a la empresa SCANIA Argentina con sede en Tucumán, ya que realizó donaciones de elementos en madera que sirvieron para la realización de dispositivos necesarios para algunas de las instancias de desarrollo y experimentación del sistema, en general, y para generar un modelo demostrativo a escala real (maqueta en 1:1).

\section{Referencias Bibliográficas}

AQUASTAT (2010) Global Water Withdrawal. sitio web AQUASTAT. Roma, Organización de las Naciones Unidas para la Alimentación y la Agricultura (FAO). www. fao.org/nr/water/aquastat/water_use/image/WithTimeNoEvap_eng.pdf.

AQUA ESPAÑA (2016) Guía Técnica de recomendaciones para el reciclaje de aguas grises en edificio. Asociación Española de Empresarios de Tratamiento y Control de Aguas.

NIF: Go8942583. No nacional en el Registro Nacional de Asociaciones: 588835. www.aquaespana.org.

Bermejo Arnaldos. D. (2012). Reutilización de aguas residuales domésticas. Estudio y comparativa de tipologías edificatorias: depuradoras naturales como alternativa sostenible. Tesis máster Arquitectura y Urbanismo Sostenible. Universitat d'Alacant. Universidad de Alicante. Canarias. 
BID-CEPAL (2018) Informe regional 2018 América Latina y El Caribe / Resumen Ejecutivo. Proceso Regional De Las Américas Foro Mundial del Agua 2018.

Daives S.; Garzón B.; Paterlini L.; Correa N.; Sancur G. (2019) Reutilización de aguas grises mediante sistema tecnológico alternativo: evaluación bacteriológica de las mismas. Revista Extensionismo, Innovación y Transferencia Tecnológica: claves para el desarrollo. P. 410:415. Facultad de Ciencias Exactas y Naturales y Agrimensura. UNNE. Corrientes. ISSN 2422-6424.

Garzón, B., Paterlini L. (2016). Vinculación Tecnológica para el Diseño y Desarrollo y Transferencia Interdiciplinardeun Sistema Tecnológico para la Reutilización de Aguas Grises.

en III Encuentro Nacional de Gestores Tecnológicos del Bicentenario, (GTec) 1 de Diciembre de 2016, Facultad de Arquitectura y Urbanismo, Universidad Nacional de Tucumán. ISBN: 978-987-754-084-0.

Gato, S., Jayasuriya, N. y Roberts, P. 2007. Temperature and rainfall thresholds for base use urban water demand modelling. Journal of Hydrology (Revista de Hidrología), Vol. 337, No. 3-4, págs. 364-376. doi.org/10.1016/j.jhydrol.2007.02.014.

Grupo de trabajo de la Comisión Sectorial de Aguas Grises. Guía Técnica Española de Recomendaciones para el Reciclaje de Aguas Grises en Edificios. Aqua España. Madrid. España.

INTI-Diseño Industrial (2015). Agua segura: una mirada de producto. Libro digital, PDF. Archivo Digital: descarga y online ISBN 978-950-532-262-6.

López Trujillo C. (2009). Manual para la reutilización de aguas grises de lavamanos en establecimientos educativos. Gobierno de Chile.

Organismo de Evaluación y Fiscalización Ambiental. (2014). Fiscalización ambiental en aguas residuales. Editorial Billy Víctor Odiaga Franco. Arequipa. Perú.

Miller, G. T.. (2003). Ciencia Ambiental. Preservemos la Tierra, Quinta Edición. Méjico. Thomson Learning.

Spiro, T. G., Stigliani, William M. (2004) “Química Medioambiental”. 2a Edición. Madrid Pearson Educación.

UNESCO, ONU-Agua (2020) Informe Mundial de las Naciones Unidas sobre el Desarrollo de los Recursos Hídricos 2020: Agua y Cambio Climático, París, UNESCO.ISBN 978-92-3-300136-7. 